\title{
A THEORETICAL MODEL FOR THE RING CURRENT INTERACTION WITH THE EARTH'S PLASMASPHERE
}

\author{
o. A. GORBACHEV \\ Irkutsk Polytechnical Institute, 664074 Irkutsk, Russia \\ G. V. KHAZANOV \\ Spacc Physics Research Laboratory, University of Michigan, 2455 Hayward, Ann Arbor, \\ MI 48109, U.S.A. \\ and \\ K. V. GAMAYUNOV and E. N. KRIVORUTSKY \\ Altai State University, 656099 Barnaul, Russia
}

(Received in final form 22 November 1991)

\begin{abstract}
This paper reports on a theoretical study of the magnetospheric ring current effect on the topside plasmasphere and ionosphere. MHD waves generated by energetic anisotropic protons of the ring current are used as the mechanism for energy transfer to plasmaspheric electrons and ions. Plasmaspheric parameters are calculated in a numerical model for ionosphere-plasmasphere coupling using a complete system of modelling equations in the 13-moment approximation of the Grad method. The calculations made have shown that the wave mechanism for energy transfer to the thermal plasma ensures its heating in the equatorial plasmasphere to experimentally observed temperatures. The resulting heat flux is able to considerably heat the plasma in the region of the topside ionosphere. It is also shown that the MHD waves present in the plasmasphere substantially influence the height profile of the electron density. The results obtained in this paper lend support to the existence of the experimentally discovered "hot" (or "warm") zone and to its influence on the underlying ionosphere.
\end{abstract}

\section{INTRODUCTION}

During the past several years our understanding of the plasmasphere has evolved through significant changes. Thus, ion temperature measurements by the Prognoz series satellites have shown that the so-called "hot", or "warm" zone exists in the plasmaspheric region adjacent to the plasmapause (Bezrukikh and Gringauz, 1976; Gringauz and Bezrukikh, 1976; Gringauz, 1983, 1985), in which the ion temperature can amount to several tens of thousands of degrees. Subsequently, the measurements reported by Gringauz were validated by experiments on the GEOS 1,2 satellites [Decreau et al., 1978, 1982 (for electrons) and Farrugia et al., 1989 (for ions)].

Immediately following the discovery of the "hot" zone Gringauz and Bezrukikh (1976) suggested that plasma heating in that zone has a wave character. Their supposition was based on the following mechanism : ring current protons generate MHD waves in the range $\omega<\omega_{b i}$ (Kennel and Petchek, 1966; Cornwall et al., 1970, 1971) which, when propagating in the region of overlap of the ring current with the plasmasphere, experience collisionless damping due to thermal particles, thereby heating them.

The earliest results regarding the heating of the cold plasma by MHD waves, were obtained by Galeev (1975). It was also ascertained that the wave heating of plasmaspheric ions occurs more effectively compared with the heating of electrons. This conclusion agrees with electron and ion temperature measurements; however, the results obtained by Galeev are an estimate and neglect the characteristic properties of the energy transfer from the waves to the charged particles of the plasmasphere as well as of the transfer processes in a plasma with increased wave activity in the lowfrequency range.

Hence, one is faced with a challenging problem of developing a mathematical model that would describe the topside plasmaspheric conditions, taking into account the active wave processes occurring there. A general scheme for such a model must include an analysis of the dispersion characteristics of the medium in order to choose suitable wave modes, a wave-particle interaction mechanism, and obtain a system of hydrodynamical equations governing macroscopic plasma parameters with proper account of the presence of an interaction such as "wave-particle". The basis for its development was formed by our earlier results (Chernov et al., 1988; Gorbachev et al., 1987, 1988, 1990a ; Konikov et al., 1989).

In view of the foregoing, the objective of this paper is to present a theoretical model for the ring currentplasmasphere interaction and to apply it, within the framework of a numerical model of ionosphereplasmasphere coupling, to calculate the main plasma- 
spheric parameters. This will make it possible to obtain space-time distributions of plasma density, temperatures and thermal fluxes as well as to determine the influence of this interaction upon the underlying ionosphere.

This paper is based on a model for ionosphereplasmasphere coupling (Gorbachev et al., 1990b, 1991) and its basic difference from the existing models implies using the more complete system of modelling equations (including the transport equation of thermal flux). That the variations in thermal fluxes should be taken into account by the model is dictated by the following reasons. Under conditions of a heated plasmasphere on L-shells adjacent to the plasmapause, the Coulomb frequency of electron collisions, $v_{e} \sim 10^{-2} \mathrm{~s}^{-1}$. Therefore, when using the Fourier law for calculating the heat flux adopted when modelling the plasmasphere and the ionosphere, errors of numerical calculation arise associated with the large value of the coefficient of electron heat conduction. This leads to the need to include a transport equation of thermal flux in the system of modelling equations.

The paper is organized as follows. Section 2 discusses mechanisms of "wave-particle" interaction for the clcctron and ion plasma components, and diffusion coefficients of electrons and ions in the wave field are obtained. The Appendix to Section 2 gives collision terms in the equations of mass, momentum and energy transport caused by the collisionless damping of the MHD waves due to thermal electrons and ions. The system of modelling equations, and the initial conditions and parameters of the model, will be presented in Section 3. Section 4 presents the results of calculations of space-time variations of the main plasma parameters in the "ionosphere-plasmasphere" system obtained in terms of a modified system of equations (in view of the "wave-particle" interaction) and of a standard system of equations (the "wave-particle" interaction is absent). These are compared for the cases of a quiet and disturbed ring current, corresponding to periods of low geomagnetic activity and of a geomagnetic storm. The main conclusions of this work are formulated in the Conclusions.

\section{THE INTERACTION OF THERMAL PLASMA WITH MHD WAVES}

The generation of MHD waves by the ring current, predicted theoretically in papers by Kennel and Petchek (1966) and Cornwall et al. $(1970,1971)$, was detected experimentally in the plasmasphere by satellites GEOS 1 and 2 (Perraut et al., 1978; Young et al., 1981 ; Rauch and Roux, 1982; Perraut, 1982;
Roux et al., 1982; Fraser, 1985). This suggests that MHD waves play the role of an intermediate link when the energy is transferred from the ring current to the plasmasphere. Measurements of the wave intensity revealed that the plasmasphere supports MHD waves of both left-handed (A waves) and righthanded polarization (FMS waves).

Because measurements have shown that in the ring current region $\left|B_{\omega}\right|^{2} \cdot \Delta \omega / B^{2} \sim 10^{-5}$ (here $\left|B_{\omega}^{2}\right| \cdot \Delta \omega$ is the energy density of the A and FMS waves, and $B$ is the Earth's magnetic field), then to obtain the integral of collisions of particles of type $\alpha$ with waves, the theory of weak turbulence can be used.

Estimates of the resonant velocities of plasmaspheric electrons and ions, together with the smallness of experimentally recorded frequencies of A and FMS waves as compared with the gyrofrequency of a proton, make it possible to choose wave-particle interaction mechanisms. For ions, this is the induced scattering off beatings of A or FMS waves, and for electrons, this is the quasilinear interaction with them (in both cases, due to Cherenkov resonance). Let us consider the two processes in greater detail.

\subsection{Quasilinear interaction of $A$ and FMS waves with background plasma electrons}

Before writing the quasilinear integral of collisions, some remarks are in order. Firstly, the interaction occurs due to Cherenkov resonance, therefore, there is no change in impulse transverse to the magnetic field. Secondly, the wavelength of A and FMS waves is of the order of $10^{7} \mathrm{~cm}$; hence the collision integral can be written in the diffusion form and, when calculating it, the spatial inhomogeneity of the magnetic field can be neglected. Thirdly, the typical time of variation of the local distribution function of electrons due to the interaction with the waves cannot be less than $1 / v_{k . l .}$, where $v_{k . l .} \sim \omega \cdot\left|B_{\omega}\right|^{2} \cdot \Delta \omega / B^{2} \cdot\left(v_{\mathrm{A}}^{2} / v_{T_{\mathrm{e}}}^{2}\right)$, which exceeds the value of $\left(\omega-k_{\|} \cdot V_{\|}\right)^{-1}$. This permits us to use the quasilinear integral in the local-time form (Akhiezer, 1974) :

$$
S_{e}^{k . l .}=\frac{\partial}{\partial v_{\|}} \cdot \sum_{\sigma} \mathbf{D}_{k .1}^{\sigma} \cdot \frac{\partial f_{\mathrm{e}}}{\partial v_{\|}},
$$

where

$$
\begin{aligned}
\mathbf{D}_{k . t .}^{\sigma}=\pi \cdot \frac{\mathrm{e}^{2}}{m_{\mathrm{e}}^{2}} \cdot \int \mathrm{d} \mathbf{k} & \cdot \frac{k_{\|}^{2} \cdot V_{\|}^{2}}{\left|g^{\sigma}\right|^{2} \cdot\left(\omega_{k}^{\sigma}\right)^{2}} \\
& \cdot\left|i \cdot E_{k_{2}} \frac{|\lambda|}{2}+E_{k_{3}} \frac{v_{\|}}{v_{\perp}}\right|^{2} \cdot \delta\left(\omega_{k}^{\sigma}-k_{\sharp} V_{\sharp}\right) .
\end{aligned}
$$

The following designations are used in (1): 
$\sigma$-wave mode ;

$\lambda=\frac{k_{\perp} \cdot V_{\perp}}{\omega_{B e}}$

$\left|g^{\sigma}\right|^{2}=1+\frac{\varepsilon_{2}^{2}}{\left(N^{2}-\varepsilon_{1}\right)^{2}}+\frac{N^{4} \cdot \sin ^{2} \theta \cdot \cos ^{2} \theta}{\left(N^{2} \cdot \sin ^{2} \theta-\varepsilon_{3}\right)^{2}}$

$N=\frac{c \cdot k}{\omega_{k}^{\sigma}}$ refractive index of wave mode $\sigma$;

$\cos \theta=\frac{k_{\|}}{k}$

$\varepsilon_{1}, \varepsilon_{2}, \varepsilon_{3}-$ components of the dielectric permittivity tensor of the cold plasma, and $\omega_{\mathrm{p} \alpha}$-plasma frequency of particles of type $\alpha$.

The expressions for the electric field components of the wave have the form:

$$
\begin{aligned}
E_{k_{2}}^{\sigma} & =-\mathrm{i} \cdot \frac{\varepsilon_{2}}{N^{2}-\varepsilon_{1}} \cdot\left|\mathbf{E}_{k}^{\sigma}\right| ; \\
E_{k_{3}}^{\sigma} & =\frac{N^{2} \cdot \sin \theta \cdot \cos \theta}{\left(N^{2} \cdot \sin ^{2} \theta-\varepsilon_{3}\right)} \cdot\left|\mathbf{E}_{k}^{\sigma}\right| ; \\
\left|\mathbf{E}_{k}^{\sigma}\right| & \equiv \mathrm{e}_{z}\left|\mathbf{E}_{k}^{\sigma}\right| .
\end{aligned}
$$

In the subsequent calculations it will be assumed that the spectral density of the wave energy does not depend on the azimuthal angle $\chi\left(\cos \chi=k_{x} / k_{\perp}\right)$, and is constant in a certain range of polar angles $\left[\theta_{1}, \theta_{2}\right] \cup\left[\pi-\theta_{2}, \pi-\theta_{1}\right], \theta_{1}, \theta_{2}<\pi / 2$.

The expression for the spectral energy density of the waves has the form:

$$
W_{k}^{\sigma}=\frac{\varepsilon_{1}}{8 \pi} \cdot\left|\mathbf{E}_{k}^{\sigma}\right|^{2},
$$

and the relation between $\left|\mathbf{E}_{k}^{\sigma}\right|^{2}$ and $\left|\mathbf{B}_{k}^{\sigma}\right|^{2}$ is given by:

$$
\begin{aligned}
&\left|\mathbf{B}_{k}^{\sigma}\right|=\frac{c^{2} \cdot k^{2}}{\left(\omega_{k}^{\sigma}\right)^{2}} \\
& \cdot\left[1-\left(\sin \theta+\mathrm{e}_{z} \cdot \cos \theta\right)^{2} \cdot \frac{1}{\left|g^{\sigma}\right|^{2}}\right] \cdot\left|\mathbf{E}_{k}^{\sigma}\right| .
\end{aligned}
$$

Since the angular dependence in the above expressions is weak, one can obtain formulas for the spectral density of electric energy of the A and FMS waves

$$
\begin{aligned}
\left|\mathbf{E}_{k}^{\mathrm{A}}\right|^{2} & =\frac{\left|B_{\omega}^{\mathrm{A}}\right|^{2} \cdot V_{\mathrm{A}}^{3} \cdot \cos ^{2} \theta_{1}^{\mathrm{A}} \cdot \cos ^{2} \theta_{2}^{\mathrm{A}}}{\omega^{2} \cdot 2 \pi \varepsilon_{1}\left(\cos ^{2} \theta_{1}^{\mathrm{A}}-\cos ^{2} \theta_{2}^{\mathrm{A}}\right)}, \\
\left|\mathbf{E}_{k}^{\mathrm{FMS}}\right|^{2} & =\frac{\left|B_{\omega}^{\mathrm{FMS}}\right|^{2} \cdot V_{\mathrm{A}}^{3}}{\omega^{2} \cdot 2 \pi \varepsilon_{1}\left(\cos ^{2} \theta_{1}^{\mathrm{FMS}}-\cos ^{2} \theta_{2}^{\mathrm{FMS}}\right)} .
\end{aligned}
$$

Since experimental values of $\left|\mathbf{B}_{\omega}^{\sigma}\right|$ have no singularities (Perraut et al., 1978; Young et al., 1981; Rauch and Roux, 1982), in our calculations $\left|\mathbf{B}_{\omega}^{\sigma}\right|$ will be con- sidered to be weakly dependent on the wave frequency in a certain range $\left[\omega_{1}^{\sigma}, \omega_{2}^{\sigma}\right]$, in which the most intensive waves are recorded.

\subsection{Induced scattering of $A$ and FMS waves due to background plasma protons}

It will be assumed here that the typical time of variation of the local distribution function of ions due to the interaction with the waves cannot be less than $1 / v_{\text {ind }}$, where $v_{\text {ind }} \sim \omega \cdot\left(\left|B_{\omega}\right|^{2} \Delta \omega / B^{2}\right)^{2} \cdot\left(v_{\mathrm{A}}^{2} / v_{T_{i}}^{2}\right)$ is the ion collision frequency with beatings of the $A$ and FMS waves. Hence it follows that for any values of ion velocities, including the interval of resonant velocities, the ion distribution function can be considered biMaxwellian.

Using a standard procedure for obtaining the collision integral in the process of induced scattering (Tsytovich, 1971 ; Livshits et al., 1987) with a longitudinal virtual wave and taking into account the remarks made when writing the quasilinear integral of collisions, one can obtain :

$$
S_{i}^{\text {ind }}=\frac{\partial}{\partial v_{1}} \cdot \sum_{\boldsymbol{\sigma}, \sigma_{1}} \mathscr{D}_{\text {ind, }, i}^{\sigma, \sigma_{1}} \cdot \frac{\partial f_{i}}{\partial v_{\|}}
$$

$$
\begin{array}{r}
\mathscr{D}_{\text {ind, }, i}^{\sigma, \sigma_{1}}=\frac{1}{m_{i}^{2}} \cdot \int \frac{\mathrm{d} \mathbf{k} \cdot \mathrm{d} \mathbf{k}_{1}}{(2 \pi)^{6}} \cdot\left|\mathbf{E}_{k}^{\sigma}\right|^{2} \cdot\left|\mathbf{E}_{k_{1}}^{\sigma}\right|^{2} \\
\cdot\left(k-k_{1}\right)_{\mid} \cdot W_{V, i}^{\sigma, \sigma_{1}}\left(k, k_{1}\right), \\
W_{V, i}^{\sigma, \sigma}\left(\mathbf{k}, \mathbf{k}_{1}\right)=\frac{(2 \pi)^{9}\left|\Lambda^{i}\left(\mathbf{k}, \mathbf{k}_{1}, \omega_{k}^{\sigma}, \omega_{k_{1}}^{\sigma}, \mathbf{V}\right)\right|^{2} \cdot 8 \pi^{4}}{\left(\omega_{k}^{\sigma}\right)^{2}} \\
\times \delta\left(\omega_{k}^{\sigma}-\omega_{k_{1}}^{\sigma}-\left(k-k_{1}\right)_{\|} v_{\|}\right)
\end{array}
$$

where:

$$
\begin{aligned}
& \Lambda^{i}\left(\mathbf{k}, \mathbf{k}_{1}, \omega_{k}^{\sigma}, \omega_{k_{1}}^{\sigma}, \mathbf{V}\right)=i \frac{\mathrm{e}_{i}^{2}}{2 g g_{1} m_{i}\left(o_{b_{i}}(2 \pi)^{3}\right.} \\
& \quad \times\left[(1+T) \cdot\left(1+T_{1}\right) \cdot \exp \left(-\mathrm{i}\left(\chi-\chi_{1}\right)\right)\right. \\
& \quad+(1-T) \cdot\left(1-T_{1}\right) \cdot \exp \left(\mathrm{i}\left(\chi-\chi_{1}\right)\right)\left\{-P^{2} \cdot \omega_{k_{1}}^{\sigma} / \omega_{b i}\right. \\
& \quad \times \frac{\left[1-J_{\mathrm{e}}\left(k_{2}\right)\right] \cdot\left(1+m_{\mathrm{e}} / m_{i}\right)}{\left[1-J_{\mathrm{e}}\left(k_{2}\right)\right]+T_{\mathrm{e}} / T_{i}\left[1-J_{i}\left(k_{2}\right)\right]}+k_{\|} k_{1 \|} / \omega_{k_{1}}^{\sigma} \cdot \omega_{b i} \\
& \left.\quad \times\left[\frac{V_{\perp}^{2}}{2}-V_{T_{i}}^{2} \cdot \frac{J_{\mathrm{e}}\left(k_{2}\right)-J_{i}\left(k_{2}\right)}{\left[1-J_{i}\left(k_{2}\right)\right]+T_{\mathrm{e}} / T_{i} \cdot\left[1-J_{\mathrm{e}}\left(k_{2}\right)\right]}\right]\right\} .
\end{aligned}
$$

Here :

$$
P=\left[1-k_{1 \|} V_{\|} / \omega_{k_{1}}^{\sigma}\right]
$$




$$
\begin{gathered}
J_{\alpha}\left(k_{2}\right) \cdot \frac{\mathrm{i}}{\sqrt{\pi} \cdot Z_{\alpha}}=\exp \left(-Z_{\alpha}^{2}\right)\left[\operatorname{sign}\left(k_{\|}-k_{1 \mathrm{H}}\right)\right. \\
\left.+2 \mathrm{i} / \sqrt{\pi} \int_{0}^{z_{\alpha}} \exp \left(t^{2}\right) \mathrm{d} t\right], \\
Z_{\alpha}=\left(\omega_{k}^{\sigma}-\omega_{k_{1}}^{\sigma_{1}}\right) /\left(k_{\|}-k_{1 \mathrm{H}}\right) / v_{T_{\alpha}} / \sqrt{2}, \\
T=\left.\frac{\varepsilon_{2}}{\left(N^{2}-\varepsilon_{1}\right)}\right|_{\omega=\omega_{k}^{\sigma}},
\end{gathered}
$$

$T_{x}$-temperature of particles of type $\alpha$.

The expressions obtained hold when $\cos \theta, \cos \theta_{1}>$ $\sqrt{m_{\mathrm{e}} / m_{i}}$

Since $v_{T_{i}} \ll v_{\mathrm{A}}$, the $\delta$-function in (6) can be expanded into a series and integrated over wave vectors. As a result, we get :

$$
\mathscr{D}_{\text {ind }}^{\mathrm{AAA}}=\frac{\chi^{\mathrm{A}, \mathrm{A}} \cdot T_{i}^{2}}{56 \cdot \rho_{i} \cdot\left(T_{i}+T_{\mathrm{e}}\right)^{2}} \cdot\left(W^{\mathrm{A}} / B^{2}\right)^{2} \cdot B^{2} \cdot\left(\bar{\omega}^{\mathrm{A}}\right)^{2} / \Delta \omega^{\mathrm{A}},
$$

$$
\begin{aligned}
& \mathscr{D}_{\text {ind }}^{\mathrm{FMS}, \mathrm{FMS}}=\frac{\chi^{\mathrm{FMS}, \mathrm{FMS}} \cdot T_{i}^{2}}{336 \cdot \rho_{i} \cdot\left(T_{i}+T_{\mathrm{e}}\right)^{2}} \\
& \times\left(W^{\mathrm{FMS}} / B^{2}\right)^{2} \cdot B^{2} \cdot\left(\bar{\omega}^{\mathrm{FMS}}\right)^{2} / \Delta \omega^{\mathrm{FMS}} \\
& \times\left(\cos ^{2} \theta_{1}^{\mathrm{FMS}}+\cos \theta_{2}^{\mathrm{FMS}} \cos \theta_{1}^{\mathrm{FMS}}+\cos ^{2} \theta_{2}^{\mathrm{FMS}}\right), \quad(10) \\
& \mathscr{D}_{\text {ind }}^{\mathrm{FMS} . \mathrm{A}}= \frac{\chi^{\mathrm{FMS}, \mathrm{A}} T_{i}^{2}}{336 \cdot \rho_{i} \cdot\left(T_{i}+T_{\mathrm{e}}\right)^{2}} \\
& \quad \times\left(W^{\mathrm{FMS}} \cdot W^{\wedge} / B^{4}\right) \cdot B^{2} \cdot \bar{\omega}^{2} \cdot \Delta \omega / \Delta \omega^{\mathrm{FMS}} / \Delta \omega^{\mathrm{A}} \\
& \quad \times\left(3+\cos ^{2} \theta_{1}^{\mathrm{FMS}}+\cos \theta_{2}^{\mathrm{FMS}} \cos \theta_{1}^{\mathrm{FMS}}+\cos ^{2} \theta_{2}^{\mathrm{FMS}}\right),
\end{aligned}
$$

where :

$$
\begin{gathered}
\rho_{i}=N_{i} \cdot m_{i}, \quad W^{\mathrm{FMS}}=\left|\vec{B}_{\omega}^{\mathrm{FMS}}\right|^{2} \cdot \Delta \omega^{\mathrm{FMS}}, \\
W^{\mathrm{A}}=\left|\bar{B}_{\omega}^{\mathrm{A}}\right|^{2} \cdot \Delta \omega^{\mathrm{A}} .
\end{gathered}
$$

In expressions (9) $-(11)\left|\bar{B}_{G(o)}^{\sigma}\right|^{2}$ and $\bar{\omega}^{\sigma}$ are certain values of the quantities $\left|B_{\omega}^{\sigma}\right|^{2}$ and $\bar{\omega}^{\sigma}$ from the interval $\left[\omega_{1}^{\sigma}, \omega_{2}^{\sigma}\right], \Delta \omega=\omega_{2}^{\sigma}-\omega_{1}^{\sigma}, \bar{\omega} \in\left[\omega_{1}^{\mathrm{A}}, \omega_{2}^{\mathrm{A}}\right] \cap\left[\omega_{1}^{\mathrm{FMS}}, \omega_{2}^{\mathrm{FMS}}\right]$ $\equiv\left[\omega_{1}, \omega_{2}\right], \Delta \omega=\omega_{2}-\omega_{1}$. The value of $\chi^{\sigma . \sigma_{1}}$ is the value of the function

$$
\frac{\left[(1+T)^{2} \cdot\left(1+T_{1}\right)^{2}+(1-T)^{2} \cdot\left(1-T_{1}\right)^{2}\right]}{g^{2} \cdot g_{1}^{2}}
$$

at points $\left(\bar{\theta}^{\sigma}, \bar{\theta}^{\sigma_{1}}\right)$ and $\left(\bar{\omega}^{\sigma}, \bar{\omega}^{\sigma_{1}}\right)$, where $\bar{\theta}^{\sigma}$ and $\bar{\theta}^{\sigma_{1}}$ are certain values of polar angles from the regions $\left[\theta_{1}^{o}, \theta_{2}^{o}\right]$ and $\left[\theta_{1}^{o}, \theta_{2}^{a}\right]$, respectively. Note that induced scattering occurs in the region where oppositelydirected wave packets exist. The diffusion coefficients of (9)-(11) are calculated for a plasma devoid of macroscopic velocities.

\subsection{Moments of collision integrals}

When calculating the moments, we shall use the inequality $\left|V_{\|}^{\mathrm{e}}-V_{\|}^{i}\right| \ll v_{T_{\mathrm{e}}}\left(V_{\|}^{\alpha}\right.$ being the macroscopic velocity of particles of type $\alpha$ ). Also, it will be assumed that the plasma distribution function is Maxwellian such that the typical scales of the irregularity are small compared with the wavelength of MHD waves.

Using a standard procedure of averaging over the phase space with weighting factors $m_{\alpha} \cdot\left(\mathbf{v}-\mathbf{V}_{\|}^{\alpha}\right)_{j}^{n}$, $n=1,2,3$, it is possible to obtain expressions for moments of the integral of collisions of the background plasma with MHD waves. Since these expressions are unwieldy in form, they are given in the Appendix.

To conclude this section, we must note the following. Quantitative estimates of the values of the formulas given in the Appendix obtained for a dipole model of the geomagnetic field, show that the collision terms caused by the presence of MHD waves in the plasmasphere have a maximum value on the equator and drop off rapidly with increasing distance from it. This is because when moving away from the equator, the plasmaspheric particles "escape" from resonance with the wave as a consequence of the increase in Alfvén velocity which is actually the phase velocity for MHD waves.

\section{THE SYSTEM OF EQUATIONS AND THE INITIAL MODEL PARAMETERS}

The model for ionosphere-plasmasphcre coupling used in this paper is based on a numerical solution of the system of magnetohydrodynamical equations for a multicomponent plasma consisting of $\mathrm{O}^{+}$- and $\mathrm{H}^{+}$ions and electrons. This does not contradict the results reported in Section 2 which were obtained for an electron-proton plasma because in the near-equatorial plasmaspheric region, where the interaction of MHD waves with electrons is the most effective, the plasma contains largely $\mathrm{H}^{+}$-ions and electrons. When writing the equations, the transverse (with respect to the geomagnetic field) plasma transport caused by external electric fields was neglected. In this case the transport processes occur along geomagnetic field lines, and all macroscopic plasma characteristics will depend only on the time $t$ and on coordinate $s$ along the geomagnetic field direction.

In view of the foregoing, the system of modelling equations in the 13-moment approximation, without taking account of the inertia of electrons and ions and of viscosity effects, can be represented as (Gorbachev et al., 1990b, 1991):

$$
\frac{\partial N_{i}}{\partial t}+\frac{1}{\sigma} \frac{\partial}{\partial s}\left(\sigma N_{i} V_{i}\right)=\frac{\delta N_{i}}{\delta t}
$$




$$
\begin{array}{r}
m_{i} N_{i} \frac{\mathrm{d} V_{i}}{\mathrm{~d} t}+\frac{\partial}{\partial s}\left(N_{i} T_{i}\right)+\frac{N_{i}}{N_{\mathrm{e}}} \frac{\partial}{\partial s}\left(N_{\mathrm{e}} T_{\mathrm{e}}\right)-m_{i} N_{i} g_{\|}=\frac{\delta V_{i}}{\delta t}, \\
i=\mathrm{H}^{+}, 0^{+} \quad(13) \\
\frac{3}{2} N_{\alpha} \frac{\mathrm{d} T_{\alpha}}{\mathrm{d} t}+\frac{5}{2} N_{\alpha} T_{\alpha} \frac{1}{\sigma} \frac{\partial}{\partial s}\left(\sigma V_{\alpha}\right)+\frac{1}{\sigma} \frac{\partial}{\partial s}\left(\sigma S_{\alpha}\right)=\frac{\delta T_{\alpha}}{\delta t}
\end{array}
$$

$$
\begin{array}{r}
\frac{\mathrm{d} S_{\alpha}}{\mathrm{d} t}+S_{\alpha}\left\{\frac{16}{5} \frac{\partial V_{\alpha}}{\partial s}+\frac{7}{5} V_{\alpha} \frac{1}{\sigma} \frac{\partial \sigma}{\partial s}\right\}+\frac{5}{2} \frac{N_{\alpha} T_{\alpha}}{m_{\alpha}} \frac{\partial T_{\alpha}}{\partial s}=\frac{\delta S_{\alpha}}{\delta t}, \\
\alpha=\mathrm{e}, i, \quad(15)
\end{array}
$$

where $\mathrm{d} / \mathrm{d} t=\partial / \partial t+V \cdot(\partial / \partial s), \sigma=B / B_{0}$ is the crosssection of the geomagnetic flux tube equal to $1 \mathrm{~cm}^{2}$ at its base $\left(B=B_{0}\right), m, N, V, T$ and $S$ are, respectively, the mass, the density, the hydrodynamical velocity along $B$ and the temperature and heat flux of charged particles, and $g_{\| \mid}$the free-fall acceleration projected onto the field line. Equation (14) for $\alpha=i$ describes space-time temperature distributions of a weightedmean ion.

The terms on the right-hand sides of equations (12)-(15) for $v_{\text {col }}>v_{\omega}$, where $v_{\omega}$ is the frequency of collisions of particles with waves, represent the variation of the respective plasma parameters caused by interparticle collisions and by collisions of particles with waves

$$
\frac{\delta}{\delta t}=\left(\frac{\delta}{\delta t}\right)_{\mathrm{coll}}+\left(\frac{\delta}{\delta t}\right)_{\omega}
$$

Terms responsible for interparticle collisions are given in papers by Schunk $(1983,1988)$, and corresponding terms that represent the interaction of electrons and ions with MHD waves are obtained in Section 2 and the Appendix. The right-hand side of the energy transport equation for the electron component also involves the plasma heating source due to photoelectrons. During the daytime, thermal electrons receive the energy from photoelectrons through direct collisions, and ions are heated through collisions with thermal electrons heated in this way. In this paper, to calculate the photoelectron source, the kinetic equation for the spectrum of the photoelectron source is solved simultaneously with the system of hydrodynamical equations (12) (15). The technique for calculating the source is similar to that suggested in papers of Khazanov (1979) and Gorbachev et al. (1991).

The system of equations was integrated along the entire length of the geomagnetic field lie. Steps in coordinate were chosen in proportion to the plasma height scale. Steps in time were varied from the value $\sim 10$ min during the morning and evening period to 1 or $2 \mathrm{~h}$ during the daytime and night-time. The boundary conditions required for solving the equations were imposed on the field line ends in the magneto-conjugate ionospheres at height $h_{0}=100 \mathrm{~km}$. At such heights, transport processes can be neglected, and the desired values can be specified from the local equilibrium condition:

$$
V_{i}\left(s_{0}, t\right)=0 ; \quad T_{\alpha}\left(s_{0}, t\right)=T_{\mathrm{n}}\left(s_{0}, t\right) ; \quad S_{\alpha}\left(s_{0}, t\right)=0,
$$

where $T_{n}$ is the temperature of the neutral atmosphere, and $s_{0}$ is the coordinate along the field line corresponding to $h_{0}$.

Time integration was performed differently for the cases of a quiet and disturbed ring current.

The case of a quiet current occurs in periods of long absence of geomagnetic disturbances and is characterized by the quiet plasmasphere with flux tubes filled with plasma, a small overlapping zone of the ring current and the plasmasphere, and by small values of the A- and FMS-wave amplitudes. It is believed that the quiet ring current is distributed approximately uniformly in longitude (Frank, 1967, 1971 ; Williams, 1981 ; Cahill, 1973). Time integration in this case was performed until a steady periodical solution with a $24-\mathrm{h}$ period was obtained. Of course, the ring current cannot retain the same intensity during such a long time interval, because it is known that geomagnetic field disturbances follow each other with a period of a few hours. Therefore, in this case a geophysical situation is investigated, which may not occur in the plasmasphere during a long time. However, only in this way can the influence of a stationary energy source of nonplasmaspheric origin upon the plasmasphere can be separated in "pure" form.

A disturbed ring current that occurs during geomagnetic storms is characterized by sharp asymmetry in longitude, a short lifetime roughly coincident with the duration of the recovery phase of the magnetic storm, and by a significant amplitude of $\mathrm{A}$ and FMS waves (Williams et al., 1973; Cahill, 1973; Williams, 1983; Frank, 1967, 1970). Time integration in this case was performed according to the following scheme. At first, plasma parameters were calculated in the absence of the "wave-particle" interaction until a steady periodical solution with a $24-\mathrm{h}$ period was obtained. The "wave-particle" interaction was then "switched-on" by a П-shaped impulse during the evening time of the day (18:00 L.T.-24:00 L.T.), which corresponded to an abrupt increase in ring current intensity during the growth phase of the storm. The nonstationary solution obtained in this way represents 
the reaction of part of the plasmasphere and of the underlying ionosphere that experienced that ring current effect, to a geomagnetic disturbance.

\section{RESULTS OF CALCULATIONS AND DISCUSSION}

Based on the calculations made, this section will present space-time distributions of the main plasma parameters in the overlapping region of the plasmasphere and the ring current in the presence of a "waveparticle" interaction. These distributions were compared with the same values ubtained using a complete system of equations (including the thermal flux transport equation), which neglects wave effects (Gorbachev et al., 1990b, 1991). The calculated values of plasma densities and temperatures are consistent with the present understanding of the Earth's ionosphere and plasmasphere and agree qualitatively with existing experimental data.

Below, we present the plasma parameter distributions in the overlapping region of the plasmasphere and the ring current for $L=5$, corresponding to equinoctial conditions and to moderate solar activity, $F_{10.7}=150$. Height profiles of the calculated plasma parameters are given from the lower boundary of the field line to the equatorial plane. On all the plots presented, the solid line represents undisturbed parameters of the plasmasphere and ionosphere obtained in Gorbachev et al. (1990b, 1991), and the dashed line refers to disturbed parameters obtained in view of the plasmaspherc-ring current interaction.

By making a correlative analysis of data from the satellite EXPLORER-45 on the mutual position of all components of the inner magnetosphere (Frank, 1971) with measurements of proton flux intensities of the ring current and its form (Frank, 1967, 1970; Williams et al., 1973, 1983), it becomes possible to obtain the following estimates of the overlapping region. During quiet periods of geomagnetic activity the region of overlap with the plasmasphere has the radial size $\approx(0.5-1) R_{\mathrm{E}}$, which does not change along the longitudinal coordinate. During a geomagnetic disturbance the radial size of the overlapping region increases to $2 R_{E}$, the ring current is characterized by strong longitudinal asymmetry, and its intensity increases several times. The typical decay time of an intensive ring current is roughly equal to the duration of the storm recovery phase $(\approx 10 \mathrm{~h})$. This leads to the fact that the overlapping region is a sector covering part of the night-time hours and the evening hours ( $\approx 18-24$ L.T., where L.T. is local time).

\subsection{Quiet ring current}

Since the model equations do not involve the internal electric field (which corresponds to a current-free approximation, $V_{\mathrm{e}}=V_{i}=V$ ), the contribution from the "wave-particle" interaction is left only in the energy transport equation (13). Besides, by estimating the contributions of different wave modes to the righthand sides of equations (12)-(15), it was shown that the A wave makes the main contribution to the process of energy transfer from waves to particles. The contribution of FMS waves makes up $\approx(5-10) \%$ of that of the A waves, depending on the external conditions.

For the stationary solution analyzed in this section, the value of the plasma temperature at each point of the field line is determined by the balance between the wave source power and the energy downward velocity. According to equations (A.1.3) and (A.2.3), the heating source of the ion component is proportional to the square of the ratio of the energy density of the waves to that of the magnetic field, and the electron source is proportional to the same ratio to the 1st power. Since the value of $W / B_{0}^{2} \ll 1$ for external plasmaspheric conditions, the inequality $J_{e}^{(2)} \gg J_{i}^{(2)}$ must then be satisfied, which, in turn, must cause $T_{\mathrm{c}}$ to exceed $T_{i}$. However, the smaller rate of heat transfer downward along the geomagnetic field line for ions compared with clcetrons leads to the fact that the ion temperature on the equator exceeds the electron temperature. If, however, the movement is downward along the field line, then the difference between $T_{\mathrm{e}}$ and $T_{i}$ at each point must decrease because the temperature in this case is now determined by the total amount of energy contained above a given point (Khazanov, 1979). Complete equalization of the ion and electron temperatures must occur at heights where the transport processes decay and the temperature is determined from local equilibrium of the sources and particle energy sinks.

Height distributions of plasma temperatures for $L=5$ at different moments of time are shown in Fig. 1 , and the diurnal variation of electron temperature for $L=5$ in the equatorial plane and at the $1000 \mathrm{~km}$ height is presented in Fig. 2. Calculations are shown, which correspond to the minimum source power (marked by symbol "*" in Table 1). The maximum temperature on the equator for ions and electrons is, respectively, $\approx 10^{4}$ and $\approx 8500 \mathrm{~K}$.

The excess of $T_{i}$ over $T_{e}$, as has already been pointed out, occurs as a consequence of the slower heat output rate for the ion component as compared with the electron component. It is quite evident that the action of the wave source on the electron component is traceable to lower heights as compared with ions (respectively, to $200 \mathrm{~km}$ for electrons and to $600 \mathrm{~km}$ for ions), which is attributed to the different character of the interaction of electrons and ions with neutral 


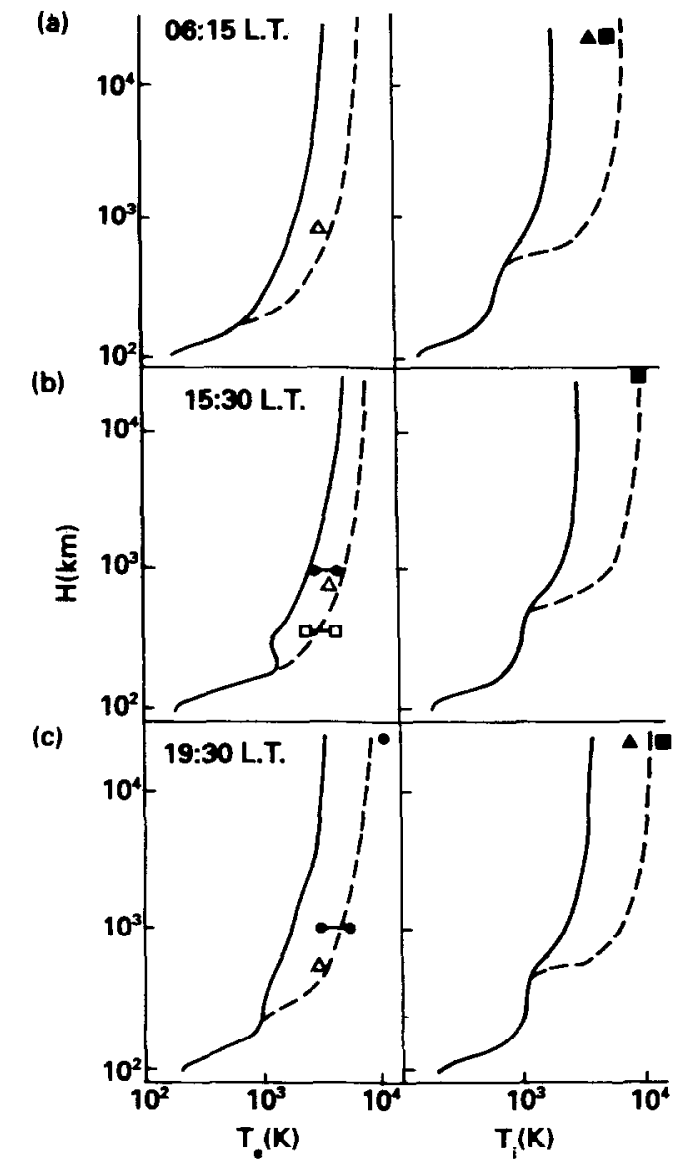

Fig. 1. Calculated distribution of electron and ION PLASMA TEMPERATURES AT THE MORNING (a), DAYTIME (b), AND EVENING (c) MOMENTS OF TIME FOR $L=5$.

Here and in Figs 2-4 and 6 the dashed and solid curves represent the plasma parameters calculated, respectively, with and without the "wave-particle" interaction taken into account.

particles of the ionosphere. At the topside ionospheric heights, $h \leqslant 1000 \mathrm{~km}$, the electron temperature amounts to $(4000-5000) \mathrm{K}$, which agrees with recent experimental data of $T_{\mathrm{e}}$ measurements at low geomagnetic activity by satellites Dynamics Explorer 1,2 (Kozura et al., 1986; Brace et al., 1987; Farrugia et al., 1989).

The ion temperature profile is characterized by large gradients $T_{i}$ at heights of $\approx 600-700 \mathrm{~km}$. This is because, at these heights, a resonant charge exchange of $\mathrm{H}^{+}$-ions with neutral hydrogen occurs, leading to a decrease of $T_{i}$ to the value of the neutral atmosphere temperature. For this same reason, the wave source effect on the ion temperature value is almost lacking (a)

(b)

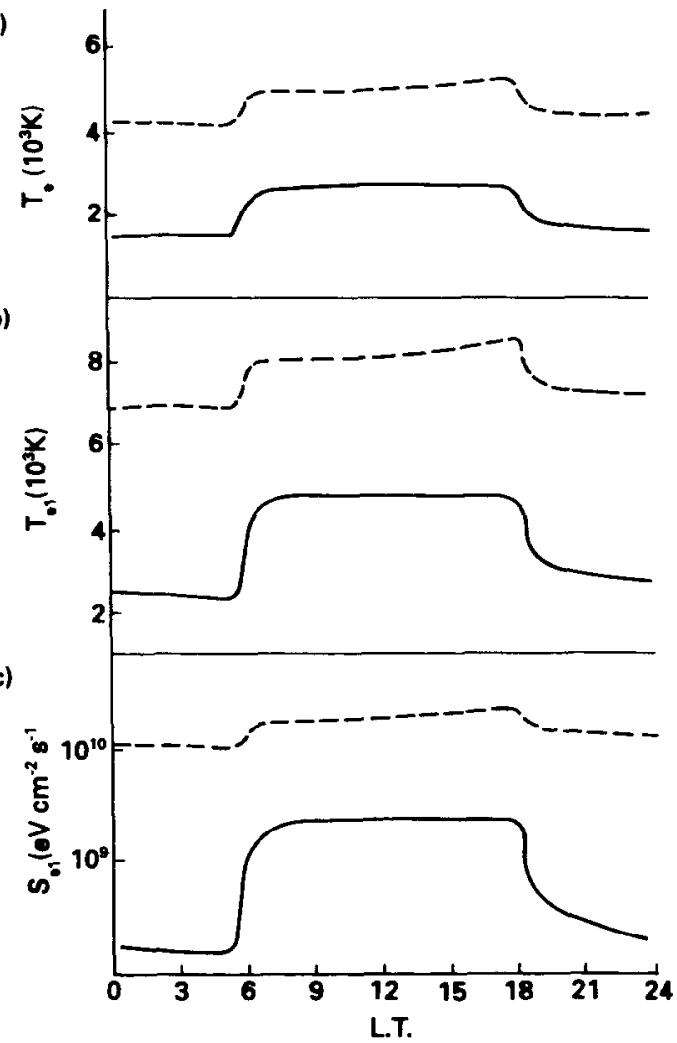

Fig. 2. Diurnal Variation of ELECtRon temperature For $L=5$ AT HEIGHT $h=1000 \mathrm{~km}$ (a) AND AT THE EQUATOR (b) AND OF THE ELECTRON HEAT FLUX AT HEIGHT $h=1000 \mathrm{~km}$ (c).

at heights $\leqslant 600 \mathrm{~km}$. The value of the electron temperature decreases rather smoothly with decreasing height because in this case no resonant processes are present, and the temperature gradient is determined

TABLE 1.

\begin{tabular}{|c|c|c|c|c|}
\hline $\begin{array}{c}\omega \\
(\mathrm{Hz})\end{array}$ & $\begin{array}{c}W^{\mathrm{A}} \\
\gamma^{2}\left(\mathrm{~Hz}^{-1}\right)\end{array}$ & $\begin{array}{c}W^{\mathrm{FMS}} \\
\gamma^{2}\left(\mathrm{~Hz}^{-1}\right)\end{array}$ & $\begin{array}{c}\theta_{1} \\
\text { (grad) }\end{array}$ & $\begin{array}{c}\theta_{2} \\
\text { (grad) }\end{array}$ \\
\hline \multirow{10}{*}{1} & \multirow{6}{*}{$0.1^{*}$} & \multirow{3}{*}{$0.01^{*}$} & $0^{*}$ & $20^{*}$ \\
\hline & & & 50 & 70 \\
\hline & & & 0 & 70 \\
\hline & & \multirow[t]{3}{*}{0.1} & 50 & 70 \\
\hline & & & 0 & 70 \\
\hline & & & 0 & 20 \\
\hline & \multirow{4}{*}{$0.5 \#$} & \multirow[t]{3}{*}{0.01} & 50 & 70 \\
\hline & & & 0 & 70 \\
\hline & & & $0 \#$ & $20 \#$ \\
\hline & & $0.1 \#$ & so & $\begin{array}{l}70 \\
70\end{array}$ \\
\hline
\end{tabular}


by the set collision frequency of the electrons with charged particles of the ionosphere.

The diurnal variation of electron temperature presented in Fig. 2, also has a number of typical features. As is quite evident from the plots, as the wave source operates, the range of temperature oscillations decreases when passing from the dayside to nightside sectors. Thus, at the equator in the absence of a wave source the electron temperature during the morning hours increases more than twice, whereas this increase of $T_{e}$ does not exceed $20 \%$ if the "wave-particle" interaction is taken into account. Such an effect is explainable by the fact that, when the wave source is taken into account, the influence of suprathermal electrons upon the time distribution of electron temperature decreases and, therefore, diurnal variations of $T_{\mathrm{c}}$ caused by "switch-on" of the photoelectron source during the daytime must decrease. For that reason, the absolute value of electron temperature calculated in view of the "wave-particle" interaction $\left(T_{\mathrm{ew}}\right)$ exceeds the electron temperature $T_{\mathrm{e} 0}$ calculated with the help of a complete system of equations (Gorbachev et al., 1991) about twice during the daytime and nearly three times during the night-time. As is apparent from the plots presented, during the daytime $T_{\text {ew }}$ increases, whereas $T_{\mathrm{e} 0}$ remains constant. This is associated with the fact that, unlike the photoelectron source that is virtually independent of the background plasma temperature, the wave source increases with increasing $T_{\mathrm{e}}$. Therefore, during the daytime, which is characterized by higher values of plasma temperature, the absolute value of the wave source increases, and this does, indeed, lead to a small increase of $T_{\mathrm{cw}}$ at $18 \mathrm{~h}$ local time (Figs 2a,b).

Differences in the space-time behaviour of thermal fluxes of electrons and ions caused by the "waveparticle" interaction are presented in Figs $2 c$ and $3 a, b$.
Figure $2 \mathrm{c}$ shows the diurnal variation of electron heat flux $S_{\text {ew }}$ calculated by taking account of the interaction of electrons with MHD waves and of heat flux $S_{\mathrm{e} 0}$, calculated from the complete system of equations (Gorbachev et al., 1991). From the plot it is evident that, as is the case with the electron temperature, diurnal variations of $S_{\mathrm{ew}}$ are much smaller than those of flux $S_{\mathrm{e} 0}$. The reason for this phenomenon, as mentioned above, is that the role of suprathermal electrons in the thermal regime of plasma decreases. The value of flux $S_{\text {ew }}$ exceeds $S_{\text {eo }}$ by about 10 times during the daytime and nearly 50 times in the night-time. The maximum value of flux $S_{\mathrm{ew}}$ is $2.5 \times 10^{10} \mathrm{eV} \mathrm{cm}^{-2} \mathrm{~s}^{-1}$ at $18 \mathrm{~h} \mathrm{L.T}$. The presence of a maximum at $18 \mathrm{~L}$.T. is attributed to the corresponding maximum in the diurnal variation of $T_{\mathrm{ew}}$.

Height profiles of ion and electron fluxes are presented in Figs 3a,b. The plots show that the wave source effect on the electron heat flux extends to smaller heights as compared with the ion flux, which is associated with the different character of the interaction of electrons and ions with ionospheric neutral particles. The greatest differences between fluxes $S_{\mathrm{cw}}$ and $S_{\mathrm{e} 0}$ and $S_{i \mathrm{w}}$ and $S_{i 0}$ correspond to heights of $\approx 600-700 \mathrm{~km}$ becausc the largest tempcrature gradients $T_{\text {ew }}$ and $T_{i w}$ are observed exactly in this height range, with rather high values of the temperatures themselves. The maximum values of heat fluxes $S_{\text {ew }}$ and $S_{i \mathrm{w}}$ are, respectively, $3 \times 10^{10}$ and $4 \times 10^{8} \mathrm{eV} \mathrm{cm}^{-2}$ $\mathrm{s}^{-1}$, which is about 50 times larger than those calculated using the complete system of equations (12)(15).

Height profiles of electron density for the morning and evening moments of time are presented in Fig. 4. The effect of the "wave-particle" interaction on plasma density is realized via the plasma temperature

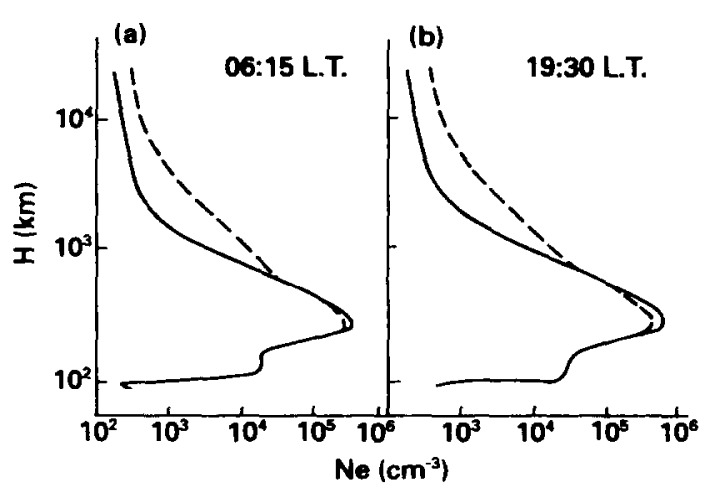

Fig. 4. Height profiles of the eleCtron density at THE MORNING (a) AND EVENING (b) MOMENTS OF TIME FOR $L=5$.
FIG. 3. HEIGHT PROFILES OF THE ELECTRON (a) AND ION (b) HEAT FLUXES DURING THE EVENING PERIOD (L.T. $=19: 30$ ) FOR

HEAT FLUXES DURING THE EVENING
\[ L=5 . \]

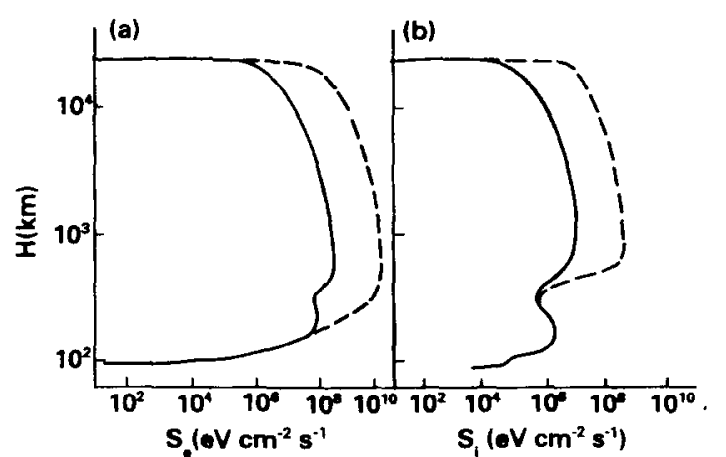


variation because the direct contribution it makes to equation (12) is zero. One can distinguish two processes which determine the difference between $N_{\text {cw }}$ and $N_{\text {e0 }}$ due to growth of $T_{\text {ew }}$ and $T_{\mathrm{iw}}$ as compared with $T_{\mathrm{e} 0}$ and $T_{10}$ :

(1) the increase of the plasma height scale in the plasmasphere; and

(2) acceleration of plasma diffusion at heights of the $\mathrm{F}_{2}$-layer maximum and the variation in production and decay reaction rate constants of the $\mathrm{O}^{+}$and $\mathrm{H}^{+}$-ions.

The second process causes part of the plasma to escape from the $F_{2}$-layer maximum to the region of higher altitudes. This effect is quite evident from the plots presented (the decrease in electron density in the $F_{2}$ layer maximum and its increase at heights of $>700$ $\mathrm{km})$. Below the $F_{2}$-layer maximum the role of the transport processes decreases, and the density is determined by the balance between the production and decay of ions of a corresponding sort. Since this process does not depend on the "wave-particle" interaction, the densities of $N_{\mathrm{ew}}$ and $N_{\mathrm{e} 0}$ become equal at heights of $<250 \mathrm{~km}$.

At plasmaspheric heights the plasma height scale has a decisive influence upon the value of electron density. Since this value increases with increasing plasma temperature, in the plasmasphere $N_{\mathrm{cw}}$ must exceed $N_{c 0}$. This is, indeed, observed on the respective plots of the height variation of electron density, and the difference between $N_{\mathrm{ew}}$ and $N_{\mathrm{e} 0}$ reaches a value in excess of $100 \%$ in the near-equatorial region of the field line.

\subsection{A disturbed ring current}

In periods of geomagnetic disturbances the ring current abruptly increases in intensity, thus leading to larger amplitude values of $\mathrm{A}$ and FMS waves in the region of its overlap with the plasmasphere. Therefore, we shall present here the results of calculations using a wave amplitude value exceeding three times the quiet level. In the table it is denoted by the symbol "\#". Since the lifetime of a disturbed ring current is short $(\approx 10 \mathrm{~h})$, the solution of the system $(12)-(15)$ with the right-hand sides of (16) will be characterized by a sharply defined nonstationarity. Thus, the typical time of plasma density variation is several days, as a consequence of which the disturbed ring current during its lifetime is unable to substantially change its space-time distribution. This is confirmed by the calculations made, according to which the plasma density variation makes up about $10 \%$ in the near-equatorial plasmaspheric region and is virtually zero at distance $R_{\mathrm{E}}$ from the field line top. Energy characteristics of plasma (temperature and heat flux) have shorter typical times, from several hours for ions to several tens of minutes for electrons and, therefore, must experience strong variations under the action of a disturbed ring current.

Height distributions of plasma temperature for $L=5$ for the evening hours (18:00 L.T.-24:00 L.T.) are presented in Fig. 5 , and diurnal variations for $T_{c}$ and $T_{i}$ for the same $L$-shell in the equatorial plane and at the $1000 \mathrm{~km}$ height are shown in Fig. 6. Owing to the different typical times of electron and ion temperature variation, the effect of the disturbed ring current on these plasma parameters is manifested differently. Thus, the time taken by the electron temperature at the equator to become steady-state is $\sim 15 \mathrm{~min}$, after that, the increase of $T_{\mathrm{c}}$ at the equator virtually ceases, and the subsequent effect of the disturbed ring current on the electron plasma component is reduced to an energy redistribution along the field line. Under the action of a strong heat flux directed downwards, the energy supplied from the ring current is transferred from the equatorial region to the topside ionospheric heights so that the electron temperature at the 1000 $\mathrm{km}$ height amounts to $\approx 6500 \mathrm{~K}$. The typical time needed by $T_{\mathrm{cw}}$ to become steady at the $1000 \mathrm{~km}$ height is about $10 \mathrm{~h}$, therefore, this temperature value is not steady-state, which is confirmed by the plots of $T_{\mathrm{ew}}$ in Figs $5 \mathrm{~b}$ and $6 \mathrm{a}$. The plots clearly show that growth of $T_{e w}$ in the equatorial plane, with the wave source switched on, virtually ceases $1 \mathrm{~h}$ after switch-on, while at $1000 \mathrm{~km}$ growth of $T_{\mathrm{ew}}$ ceases only after the source of electron heating by MHD waves is switched off. With decreasing height, the typical time taken by $T_{\text {ew }}$ to become steady-state increases, which leads to its gradual increase at the $F_{2}$-layer heights and to the appearance of differences between $T_{\mathrm{ew}}$ and $T_{\mathrm{e} 0}$ at still lower heights.

As is clearly evident from Fig. 6a, the $1000 \mathrm{~km}$ height, following switch-on of the wave source, exhibits, initially, a decrease of $T_{\text {ew }}$ and then a rapid growth to the value of $\approx 4000 \mathrm{~K}$. This is associated with the fact that the electron heating in the topside ionospheric region occurs due to energy input from the region of wave source operation and, consequently, the beginning of heating will be delayed by time $\Delta t \sim \mathscr{L} / v_{r_{\mathrm{c}}}$, where $\mathscr{L}$ is the length of a portion of the field line from the equator to the topside ionospheric heights. A temperature decrease during the period from 18:00 L.T. (the time of wave source "switch-on") to $(18: 00+\Delta t)$ L.T. is caused by "switchoff" of the photoelectron plasma heating source at 18:00 L.T.

The typical time taken by the ion temperature to reach a steady-state value is $\approx 10 \mathrm{~h}$, which leads to a 

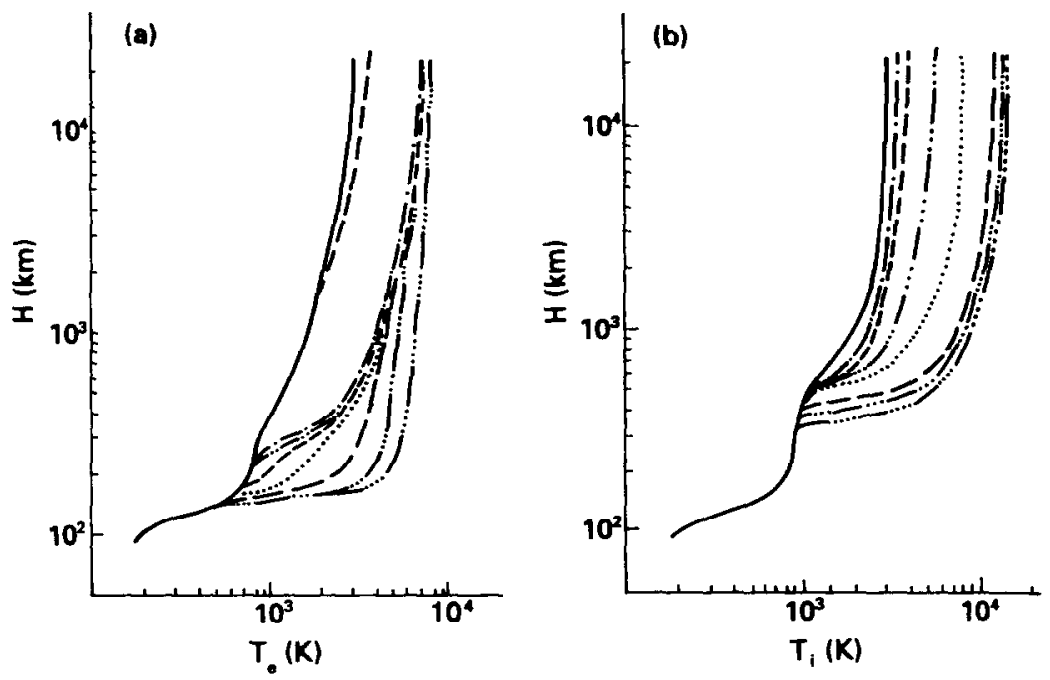

Fig. 5. SPACF Distributions of EI.ECTRON (a) AND ION (b) TEMPERATURES AT DIFFFrFNT MOMENTS OF TIME FOLLOWING RING CURRENT "SWITCH-ON" FOR $L=5$.

The curves correspond to the following moments of time :

18:00 L.T.; - — 18:05 L.T. ; - . - 18:15 L.T.; - - 18:45 L.T.; - . - 19:45 L.T.; $\cdots \cdots$ 21:00 L.T. ; - 22:30 L.T.; - -.. 23:15 L.T.; - - . 00:15 L.T.

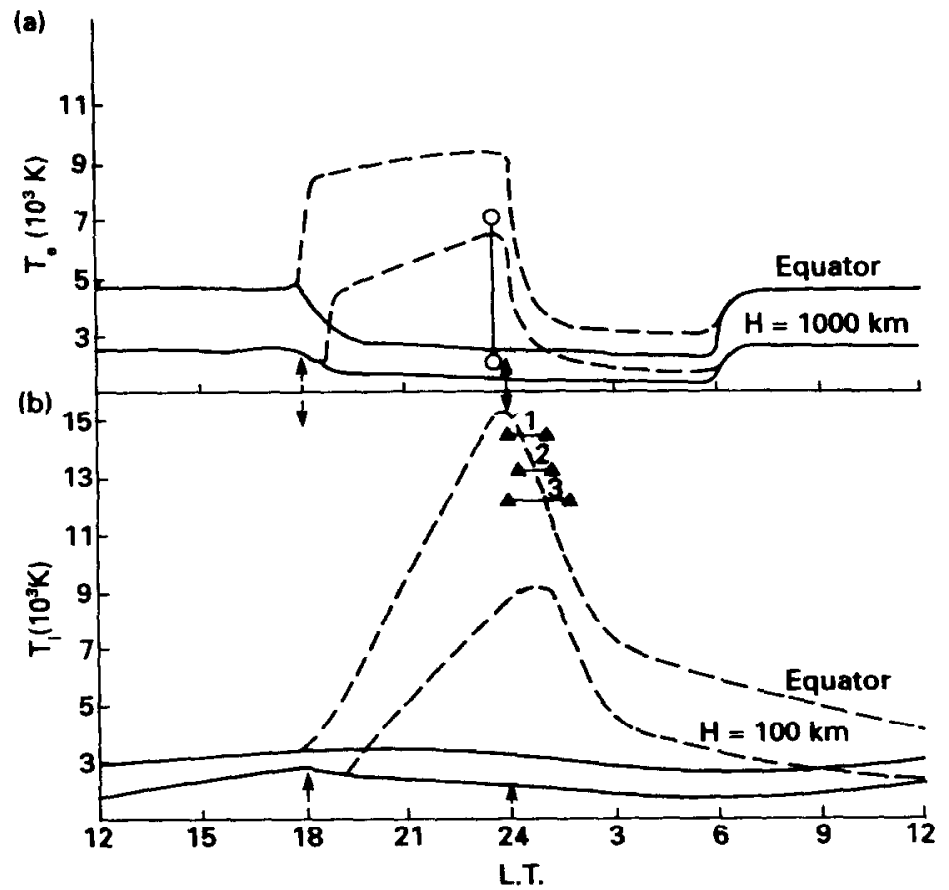

Fig. 6. Diurnal variation of The electron (a) and iOn (b) TfMperature at $L=5$ for the CaSE of a DISTURBED RING CURRENT.

The arrows indicate the time of wave source "switch-on" and "switch-off". 
smooth increase of $T_{i \mathrm{w}}$ during the entire time of the wave source operation (see Figs $5 \mathrm{~b}$ and $6 \mathrm{~b}$ ). As is quite evident from the plots in Fig. $5 b$, during the initial period of source operation the ion temperature increases weakly, and the height profile of $T_{\text {iw }}$ is characterized by rather large gradients on the plasmaspheric portion of the field line. However, with increasing equatorial value of ion temperature, the absolute value of the ion source (A.2.3) increases, which leads to a faster growth of $T_{i w}$ to values of $\approx 1.5 \times 10^{4} \mathrm{~K}$. The excess of values of $T_{i w}^{\mathrm{eq}}$ over $T_{\mathrm{ew}}^{\mathrm{eq}}$, as mentioned above, is attributable to the slower heat escape from the interaction region for the ion component. The subsequent time evolution of height distribution of the ion temperature is reduced to energy transfer from the heating region to the topside ionosphere, and this process occurs far more slowly than for the case of the electron component. Therefore, the height profile of $T_{i w}$ is characterized by larger gradients than that of $T_{\mathrm{ew}}$ at the moment of time preceding "switch-off" of the wave source. The diurnal variation of ion temperature at $1000 \mathrm{~km}$, as in the case of the electron component, is characterized by a delay of temperature increase relative to the time of wave source "switch-on". The value of delay, $\Delta t \sim \mathscr{L} / V_{i}$, as is clearly seen in Fig. 6, is large compared with the electron case because $V_{i} \ll v_{T_{\mathrm{c}}}$. The decrease of the value of $T_{i w}$ during 18:00-(18:00+ $\left.\Delta t\right)$ L.T. is accounted for by the same factor as in the case of the electron component. The maximum value reached by the ion temperature at $1000 \mathrm{~km}$ is $\approx 8000$ $\mathrm{K}$. The plots in Fig. $5 \mathrm{~b}$ clearly show that the height profile of $T_{\text {iw }}$ does not reach a steady-state level throughout the length of the field line. As has already been pointed out, this is because the typical time taken by the ion temperature to reach a steady-state value roughly coincides with the duration of the wave source operation. It should be noted that in the case of the ion component the effect of the influence on the value of $T_{i w}$ of the wave source following its switch-off is present. As is evident from the plots presented, excess values of ion temperature occur $\Delta t \approx 10 \mathrm{~h}$ after the disturbed ring current ceases its action on the plasma. The same effect is observed at the topside ionospheric heights as well.

The available experimental data on electron and ion temperature variations are presented in Figs 1 and 6 according to those reported by Decreau et al. (1982), Gringauz (1985), Kozura et al. (1986), Brace et al. (1987, 1988), and Farrugia et al. (1989). The following designations are used in the figures:

- -electron temperature measurements at the equator obtained by the satellite GEOS 1 (Decreau $e t$ al., 1982); $\triangle$-ion temperature measurements at the equator obtained by the satellite GEOS 1 (Farrugia et al., 1989), 1,2,3-respectively, days $137\left(\Sigma K_{p}=17\right), 139$ $\left(\Sigma K_{p}=14\right)$ and $136\left(\Sigma K_{p}=9\right)$ of 1989 ;

-ion temperature measurements at the equator obtained by the satellites Prognoz 1,2 (Gringauz, 1985); and

$0, \triangle, \square$-electron temperature measurements at ionospheric heights obtained by the satellite Dynamics Explorer 2 and presented, respectively, in papers by Brace et al. $(1987,1988)$ and Kozura et al. (1986).

One can see in Fig. 1 that geomagnetically-quiet periods show rather good agreement between experiment and calculations. However, it is difficult to speak of a quantitative comparison of the results in this case because of the insufficient number of experimental points on the curves.

The disturbed ring current conditions (Fig. 6) also exhibit qualitative agreement between the results of the calculations with electron and ion temperature measurements presented by Kozura et al. (1986) and Farrugia et al. (1989). Also in this case, however, the small number of electron and ion temperature measurements does not permit us to draw any conclusions about some quantitative characteristics of the comparison. Besides, in this paper the regime of a disturbed ring current is introduced from the amplitude value of MHD waves generated by it and is unassociated with the $K_{p}$-index variation, which also makes a quantitative comparison of calculations with experiment difficult.

A somewhat different picture emerges when ion temperature measurements obtained by the satellites Prognoz 1,2 are used (Bezrukikh and Gringauz, 1976; Gringauz and Bezrukikh, 1976; Gringauz, 1983, 1985). Results of these measurements give $T_{i}=(25$ $50) \times 10^{3} \mathrm{~K}$, while according to the results of calculations the ion temperature does not exceed $\approx 15,000 \mathrm{~K}$. Hence, it follows that not all mechanisms that control the plasma thermal regime of the "ionosphere-plasmasphere" system are included in this model. One of such mechanisms can be the influence on plasma transfer coefficients (in particular, on the heat conduction coefficient) of current plasma instabilities at ionospheric heights (Ionson et al., 1976, 1979; Okuda et al., 1981). However, a proper account of such an additional plasma heating source in the equatorial zone of the plasmasphere is beyond the scope of the present study.

To conclude, we wish to note the following. In the calculations it was assumed that the duration of ring current decay during geomagnetic disturbances is about $10 \mathrm{~h}$. In the real geophysical situation the ring 
current can decay in a much longer time; however, the calculations have shown that no qualitative changes in space-time variations of the main plasma parameters occur in this case.

\section{CONCLUSIONS}

In this paper we have presented a model for ionosphere-plasmasphere coupling which includes the effect of $A$ and FMS waves generated by the ring current on plasmaspheric electrons and ions. The equations are integrated along the entire length of the field line between the conjugate ionospheres in the nonstationary approximation. We have obtained space-time distributions of the main plasma parameters for the cases of a quiet ring current (low geomagnetic activity) and a disturbed ring current (geomagnetic storm). The results obtained permit us to draw the following conclusions.

\subsection{Quiet ring current}

(1) A quiet ring current, corresponding to geomagnetically-quiet periods, is able to heat the plasmasphere to temperatures exceeding undisturbed values by a factor of two during the daytime and three times at night. The absolute values of plasma temperature at the equator are $\approx 8500 \mathrm{~K}$ for electrons and $\approx 10,000$ $\mathrm{K}$ for ions. At the topside ionospheric heights $(\approx 1000$ km) $T_{\mathrm{e}} \approx 4500 \mathrm{~K}$ and $T_{i} \approx 5500 \mathrm{~K}$.

(2) The ion temperature always exceeds the electron temperature in the plasmasphere and topside ionosphere. At low heights $(h \approx 500 \mathrm{~km}) T_{\mathrm{e}}>T_{i}$.

(3) The diurnal variation of electron temperature, when the "wave-particle" interaction is taken into account, is characterized by the absence of abrupt fluctuations in going from the daytime to the nighttime, typical of the undisturbed case.

(4) Heat fluxes are able to amount to $\approx 3 \times 10^{10} \mathrm{eV}$ $\mathrm{cm}^{-2} \mathrm{~s}^{-1}$ for electrons and $\approx 4 \times 10^{8} \mathrm{eV} \mathrm{cm}^{-2} \mathrm{~s}^{-1}$ for ions, which exceeds more than 10 times the undisturbed values of fluxes during the daytime and nearly 50 times at night.

(5) There is a significant variation in the height profile of electron density, particles are transferred from the $\mathrm{F}_{2}$-region maximum to the topside ionospheric heights due to an increase in diffusion and to a change of reaction rate constants of production and decay of $\mathrm{O}^{+}-$and $\mathrm{H}^{+}$-ions, and there is a considerable increase in particle density on the plasmaspheric portion of the field line caused by the increase in plasma height scale.

\subsection{Disturbed ring current}

(1) The space-time distributions of plasma parameters obtained for the regime of a disturbed ring current are characterized by a sharply expressed nonstationarity. During an $8 \mathrm{~h}$ heating the plasma temperature reaches these values : at the equator $\approx 9500$ $\mathrm{K}$ for electrons and $\approx 15,000 \mathrm{~K}$ for ions; at height $h \approx 1000 \mathrm{~km}, \approx 6500 \mathrm{~K}$ for electrons and $\approx 9000 \mathrm{~K}$ for ions. In this case, only the equatorial electron temperature reaches a steady-state (stationary) level.

(2) At ionospheric heights, plasma heating starts with a delay with respect to the moment of ring current "switch-on". This delay is determined by the height and rate of energy sink along the field line. At height $h=1000 \mathrm{~km}$ it is $10 \mathrm{~min}$ and $\approx 1 \mathrm{~h}$ for electrons and ions, respectively.

(3) Following cessation of the disturbed ring current effect on the plasma, the ion temperature is still influential for about $10 \mathrm{~h}$. The electron temperature returns to its undisturbed value $\mathrm{l}$ or $2 \mathrm{~h}$ after source "switch-off".

(4) The influence of the $8 \mathrm{~h}$ long effect of the disturbed ring current on plasma density implies a $10 \%$ deviation of the density from its undisturbed level within a rather narrow equatorial region of the plasmasphere.

(5) The comparison of the results of the calculations made in this section qualitatively agree with the experimental plasma temperature measurements in the plasmasphere and the ionosphere. One cannot speak of some quantitative characteristics of the comparison in this case because the number of plasma temperature measurements in the ionosphere and the plasmasphere is insufficient.

Acknowledgements-We are grateful to Dr Yu. V. Konikov (IZMIRAN, U.S.S.R.) for helpful discussions, and to $\mathrm{Mr}$ V. G. Mikhalkovsky (SibIZMIR, U.S.S.R.) for his assistance in preparing the English version of the manuscript and for typing the text.

\section{REFERENCES}

Akhiezer, A. I. (Ed.) (1974) Plasma Electrodynamics. Nauka, Moscow,

Bezrukikh, V. V. and Gringauz, K. I. (1976) The hot zone in the outer plasmasphere of the Earth. J. atmos terr. Phys. 38. 1085 .

Brace, L. H., Chappel, C. R., Chandler, M. O., Comfort, R. H., Horwitz, J. L. and Hoegy, W. R. (1988) F region electron temperature signatures of the plasmapause based on Dynamics Explorer 1 and 2 measurements. J. geophys. Res. A93, 1896.

Brace, L. H., Theis, R. F. and Hoegy, W. R. (1987) Ionospheric electron temperature at solar maximum. $A d v$. Space Res. 7(6), 99.

Cahill, L. J. (1973) Magnetic storm inflation in the evening sector. J. geophys. Res. 78, 4724.

Chernov, A. A., Khazanov, G. V. and Konikov, Yu. V. (1988) Linear interaction of Alfvén and magnetosound 
waves on Cherenkov resonance with ring current's and plasmasphere's particles. Preprint No. 34 (788), p. 26, IZMIRAN, Moscow.

Cornwall, J. M., Coroniti, F. V. and Torn, R. M. (1970) Turbulent loss of ring current protons. J. geophys. Res. 75, 4699.

Cornwall, I. M., Coroniti, F. V. and Torn, R. M. (1971) Unified theory of SAR-arc formation at the plasmapause. J. geophys. Res. 76, 4428.

Decreau, P. M. E., Beghin, C. and Parret, M. (1978) Electron density and temperature, as measured by the mutial impedance experiment on board GEOS-1. Space Sci. Rev. 22, 581 .

Decreau, P. M. E., Beglin, C. and Parret, M. (1982) Global characteristics of the cold plasma in the equatorial plasmasphere region as deduced from the GEOS-1 mutial impedance probe. I. geophys. Res. A87, 695.

Farrugia, C. J., Young, D. T. and Geiss, J. (1989) The composition, temperature and density structure of cold ions in the quiet terrestrial plasmasphere. J.geophys. Res. A94, 11865.

Frank, L. A. (1967) On the extraterrestrial ring current during geomagnetic storms. J. geophys. Res. 72, 3753.

Frank, L. A. (1970) Direct detection of asymmetric increases of extraterrestrial RC protons intensities in the outer radiation zone. J.geophys. Res. 75, 1263.

Frank, L. A. (1971) Relationship of the plasma sheet, ring current, trapping boundary and plasmapause near the magnetic equator and local midnight. J. geophys. Res. 76, 2265 .

Fraser, B. J. (1985) Observation of ion cyclotron waves near synchronous orbit and on the ground. Space Sci. Rev. $\mathbf{4 2}$, 357.

Galeev, A. A. (1975) Plasma turbulence in the magnetosphere with special regard to plasma heating, in Physics of the Hot Plasma in the Magnetosphere (Edited by Hultquist, B. and Stenfio, L.), p. 251. Plenum Press, New York/ London.

Gorbachev, O. A., Konikov, Yu. V. and Khazanov, G. V. (1987) Quasilinear heating of thermal electrons for interaction plasmasphere with ring current. Geomagnetism $i$ aeronomiya 27,625 .

Gorbachev, O. A., Konikov, Yu. V. and Khazanov, G. V. (1988) Quasilinear heating of clcctrons in the Earth's plasmasphere. Pure Appl. Geophys. 127, 545.

Gorbachev, O. A., Konikov, Yu. V. and Khazanov, G. V. (1990a) The transport equation for thermal electrons in the region of interaction plasmasphere with ring current. Geomagnetizm i aeronomiya 30, 200.

Gorbachev, O. A., Konikov, Yu. V., Sidorov, I. M. and Khazanov, G. V. (1990b) The calculation of electron temperature in the system "ionosphere-plasmasphere". Geomagnetizm i aeronomiya 30, 495.

Gorbachev, O. A., Konikov, Yu. V., Sidorov, I. M. and Khazanov, G. V. (1991) Allowance for thermal flux variations in the model of ionosphere-plasmasphere interactions. Planet. Space Sci. 39, 847.

Gringauz, K. I. (1983) Plasmasphere and its interaction with ring current. Space Sci. Rev. 34, 245.

Gringauz, K. I. (1985) Structure and properties of the Earth plasmasphere. Adv. Space Res. 5, 391.

Gringauz, K. I. and Bezrukikh, V. V. (1976) Earth's plasmasphere. Geomagnetizm i aeronomiya 17, 784.

Ionson, J. A., Ong, R. S. B. and Fontheim, E. G. (1976) Anomalous resistivity of the auroral plasma of the topside ionosphere. Geophys. Res. Lett. 3, 549.
Ionson, J. A., Ong, R. S. B. and Fontheim, E. G. (1979) Turbulent transport and heating in auroral plasma of the topside ionosphere. Planet. Space Sci. 27, 203.

Kennel, C. F. and Petchek, H. E. (1966) Limit on stable trapped particle fluxes. J. geophys. Res. 71, 1.

Khazanov, G. V. (1979) Kinetics of the Electron Component of the Upper Atmosphere Plasma (in Russian). Nauka, Moscow.

Konikov, Yu. V., Gorbachev, O. A., Khazanov, G. V. and Chernov, A. A. (1989) Hydrodynamics equation for thermal electrons taking into account their scattering on ioncyclotron waves in the outer plasmasphere of the Earth. Planet. Space Sci. 37, 1157.

Kozura, J. U., Brace, L. II., Gravens, T. E. and Nagy, A. F. (1986) A statistical study of the subauroral electron temperature enhancement using Dynamics Explorer 2 langmuir probes observation. J. geophys. Res. A91, 11270.

Livshits, M. A. et al. (1987) Fizika plasmy 13, 303.

Okuda, H., Cheng, C. Z. and Lee, W. W. (1981) Numerical simulation of electrostatic hydrogen cyclotron instabilities. Physics Fluids 24, 1060.

Perraut, S. (1982) Waves-particle interactions in the ULF range : GEOS 1 and 2 results. Planet. Space Sci. 30, 1219.

Perraut, S., Gendrin, R., Roux, A. and de Villiedary, C. (1978) J. geophys. Res. 82, 2314.

Rauch, J. L. and Roux, A. (1982) Ray tracing of ULF waves in a multicomponent magnetospheric plasma: consequences for generation mechanism of ion cyclotron waves. J. geophys. Res. 87, 8191.

Roux, A., Perraut, S., Rauch, J. L., et al. (1982) Waveparticle interactions near $\Omega_{\mathrm{He}^{+}}$observed on board GEOS 1 and 2. 2. Generation of ion cyclotron waves and heating $\mathrm{He}^{+}$ions. J. geophys. Res. 87, 8174.

Schunk, R. W. (1983) Solar-Terrestrial Physics (Edited by Carovilliano, R. L. and Forbes, J. M.), p. 609. D. Reidel, Hingham, MA.

Schunk, R. W. (1988) A mathematical model of the middle and high latitude ionosphere. Pure Appl. Geophys. 127, 255.

Tsytovich, V. N. (1971) Theory of Turbulent Plasma (in Russian). Atomizdat, Moscow.

Williams, D. J. (1981) Ring current composition and source : an update. Planet. Space Sci. 29, 1195.

Williams, D. J. (1983) The Earth's ring current: causes, generation, and decay. Space Sci. Rev. 34, 223.

Williams, D. J., Fritz, T. A. and Conradi, A. (1973) Observation of proton spectra $\left(1.0<E_{p}<300 \mathrm{keV}\right)$ and particle fluxes at the plasmapause. J. geophys. Res. 78, 4751 .

Young, D. T., Perraut, S., Roux, A., de Villedary, C., Gendrin, R., Korth, A., Kremser, G. and Jones, D. (1981) Wave-particle interactions near $\mathrm{He}^{+}$observed on GEOS 1 and 2. I. Propagation of ion cyclotron waves in $\mathrm{He}^{+}$rich plasma. J. geophys. Res. 86, 6755.

\section{APPENDIX}

\section{A.1. Interaction "electron-A wave"}

$$
J^{(0)}=0
$$

$$
J_{\alpha}^{(1)}=\delta_{\alpha 3} \frac{\left(\beta^{c} V_{\AA}^{2}\right)^{3 / 2}}{(2 \pi)^{1 / 2}} \cdot \rho_{e} L_{1}\left[A_{1}+2 \cdot \frac{V_{T_{e}}^{2}}{V_{\Lambda}^{2}} A_{2}+8 \cdot \frac{V_{T_{e}}^{4}}{V_{\Lambda}^{4}} A_{5}\right]
$$

$$
\alpha=1,2,3 \text {. }
$$




$$
\begin{gathered}
J_{\alpha \beta}^{(2)}=2 \cdot \delta_{\alpha 3} \delta_{\beta 3} \frac{\left(\beta^{e} V_{\mathrm{A}}^{2}\right)^{3 / 2}}{(2 \pi)^{1 / 2}} \cdot \rho_{\mathrm{e}} L_{2}\left[A_{1}+2 \cdot \frac{V_{T_{\mathrm{c}}}^{2}}{V_{\mathrm{A}}^{2}} A_{2}+8 \cdot \frac{V_{T_{\mathrm{c}}}^{4}}{V_{\mathrm{A}}^{4}} A_{3}\right] . \\
\alpha, \beta=1,2,3 . \quad(\mathrm{A} .1 .3) \\
J_{321}^{(3)}=0, \quad J_{\alpha \beta \gamma}^{(3)}=0, \quad \alpha, \beta, \gamma=1,2 . \quad(\mathrm{A} .1 .4) \\
J_{3 \alpha \alpha}^{(3)}=\frac{\left(\beta^{c} V_{\mathrm{A}}^{2}\right)^{3 / 2} \cdot v_{T_{\mathrm{e}}}^{2}}{(2 \pi)^{1 / 2}} \cdot \rho_{\mathrm{e}} L_{1}\left[A_{1}+4 \cdot \frac{V_{T_{\mathrm{e}}}^{2}}{V_{\mathrm{A}}^{2}} A_{2}+24 \cdot \frac{V_{T_{\mathrm{e}}}^{4}}{V_{\mathrm{A}}^{4}} A_{3}\right] . \\
J_{333}^{(3)}=3 \cdot \frac{\left(\beta^{\mathrm{c}} V_{\mathrm{A}}^{2}\right)^{3 / 2}}{(2 \pi)^{1 / 2}} \cdot \rho_{\mathrm{e}} L_{3}\left[A_{1}+2 \cdot \frac{V_{T_{\mathrm{c}}}^{2}}{V_{\mathrm{A}}^{2}} A_{2}+8 \cdot \frac{V_{T_{\mathrm{c}}}^{4}}{V_{\mathrm{A}}^{4}} A_{3}\right] .
\end{gathered}
$$

$$
\begin{aligned}
& A_{1}=\left.R \cdot\left\{\eta^{2} \cdot \ln y+\eta^{3} \cdot \frac{1}{y} \cdot\left(1+\frac{1}{2 y}\right)+2 \cdot \eta^{5 / 2} \operatorname{arct} g-\frac{y}{\sqrt{\eta}}\right\}\right|_{\theta_{1}^{\prime}} ^{p_{2}^{A}}, \\
& A_{2}=\left.2 R \cdot\left\{\eta^{2} \cdot\left(-\ln y+\frac{1}{y}\right)+\eta y+\eta^{2} \ln \left(\eta+y^{2}\right)\right\}\right|_{\mid \theta_{1}^{\hat{2}}} ^{\theta_{2}^{A}}, \\
& A_{3}=\left.R \cdot\left\{\eta \cdot(-\ln y+y)+y^{2} / 2-2 \cdot \eta^{3 / 2} \operatorname{arctg} \frac{y}{\sqrt{\eta}}\right\}\right|_{\theta_{1}^{A}} ^{\theta_{2}^{A}}, \\
& R=\frac{\pi \cdot \bar{\omega}^{\mathrm{A}} \cdot\left|\bar{B}_{\omega}^{\mathrm{A}}\right|^{2} \cdot \Delta \omega^{\mathrm{A}} \cdot \cos ^{2} \theta_{1}^{\mathrm{A}} \cdot \cos ^{2} \theta_{2}^{\mathrm{A}}}{16 \cdot B^{2} \cdot\left(\cos ^{2} \theta_{1}^{\mathrm{A}}-\cos ^{2} \theta_{2}^{\mathrm{A}}\right)}, \\
& \eta=4 \cdot\left(\frac{\bar{\omega}^{\mathrm{A}}}{\omega_{b i}}\right)^{2}, \quad y=\sqrt{\sin ^{4} \theta+\eta \cdot \cos ^{2} \theta}-\sin ^{2} \theta,
\end{aligned}
$$

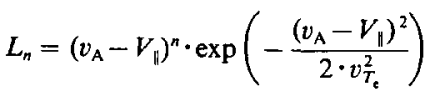

$$
\begin{aligned}
& +(-1)^{n} \cdot\left(v_{\mathrm{A}}+V_{\|}\right)^{n} \cdot \exp \left(-\frac{\left(v_{\mathrm{A}}+V_{\|}\right)^{2}}{2 \cdot v_{T_{\mathrm{e}}}^{2}}\right), \\
& V_{\|}=V_{\|}^{\mathrm{e}}-V_{\|}^{i}, \quad \beta^{\alpha}=m_{\alpha} / T_{\alpha} .
\end{aligned}
$$

\section{A.2. Interaction "electron-FMS wave"}

$$
\begin{aligned}
& J^{(0)}=0 . \\
& J_{a}^{(1)}=\delta_{\alpha 3} \frac{\left(\beta^{e} V_{\mathrm{A}}^{2}\right)^{3 / 2}}{(2 \pi)^{1 / 2}} \cdot \rho_{\mathrm{e}} \int \mathrm{d} v_{\mathfrak{\natural}} \cdot \exp \left(-\frac{v_{\|}^{2}}{2 \cdot v_{T_{\mathrm{e}}}^{2}}\right) \\
& \times v_{\|}\left[A_{1}+2 \cdot \frac{V_{T_{\mathrm{c}}}^{2}}{V_{\mathrm{A}}^{2}} \cdot A_{2}+8 \cdot \frac{V_{T_{\mathrm{c}}}^{4}}{V_{\mathrm{A}}^{4}} \cdot A_{3}\right], \\
& \frac{v_{\mathrm{A}}}{\cos \theta_{1}^{\text {FMS }}} \leqslant\left|v_{\sharp}+V_{\|}\right| \leqslant \frac{v_{\mathrm{A}}}{\cos \theta_{2}^{\text {FMS }}}, \quad \alpha=1,2,3 .
\end{aligned}
$$

$$
\begin{aligned}
J_{\alpha \beta}^{(2)}=2 \delta_{\alpha 3} \delta_{\beta 3} \cdot & \frac{\left(\beta^{e} V_{\mathrm{A}}^{2}\right)^{3 / 2}}{(2 \pi)^{1 / 2}} \rho_{\mathrm{c}} \int \mathrm{d} v_{\|} \cdot \exp \left(-\frac{v_{\|}^{2}}{2 \cdot v_{T_{\mathrm{c}}}^{2}}\right) \\
& \times v_{\|}^{2}\left[A_{1}+2 \cdot \frac{V_{T_{\mathrm{c}}}^{2}}{V_{\mathrm{A}}^{2}} \cdot A_{2}+8 \cdot \frac{V_{T_{\mathrm{c}}}^{4}}{V_{\mathrm{A}}^{4}} \cdot A_{3}\right],
\end{aligned}
$$

$$
J_{3 \alpha \alpha}^{(3)}=v_{T_{\mathrm{e}}}^{2} \cdot \frac{\left(\beta^{\mathrm{c}} V_{\mathrm{A}}^{2}\right)^{3 / 2}}{(2 \pi)^{1 / 2}} \rho_{\mathrm{e}} \int \mathrm{d} v_{\|} \cdot \exp \left(-\frac{v_{\|}^{2}}{2 \cdot v_{T_{\mathrm{e}}}^{2}}\right)
$$$$
\times v_{\mathrm{h}}\left[A_{1}+4 \cdot \frac{V_{T_{\mathrm{c}}}^{2}}{V_{\mathrm{A}}^{2}} \cdot A_{2}+24 \cdot \frac{V_{T_{\mathrm{c}}}^{4}}{V_{\mathrm{A}}^{4}} \cdot A_{3}\right],
$$$$
J_{321}^{(3)}=0, \quad J_{\alpha \beta \gamma}^{(3)}=0, \quad \alpha, \beta, \gamma=1,2 .
$$

$$
\begin{array}{r}
J_{333}^{(3)}=3 \cdot \frac{\left(\beta^{\mathrm{e}} V_{\mathrm{A}}^{2}\right)^{3 / 2}}{(2 \pi)^{1 / 2}} \rho_{\mathrm{e}} \int \mathrm{d} v_{\|} \cdot \exp \left(-\frac{v_{1}^{2}}{2 \cdot v_{T_{\mathrm{c}}^{2}}^{2}}\right) \\
\quad \times v_{\|}^{3}\left[A_{1}+2 \cdot \frac{V_{T_{\mathrm{c}}}^{2}}{V_{\mathrm{A}}^{2}} \cdot A_{2}+8 \cdot \frac{V_{T_{\mathrm{c}}^{4}}^{4}}{V_{\mathrm{A}}^{4}} \cdot A_{3}\right], \quad(\mathrm{A} .2 .6) \\
A_{2}=2 \cdot R \cdot Q, \quad A_{1}=\frac{A_{2}^{2}}{4 \cdot R}, \quad A_{3}=R, \eta=4 \cdot\left(\frac{\bar{\omega}^{\mathrm{FMS}}}{\omega_{b i}}\right)^{2}, \\
R=\frac{\pi \cdot\left(\bar{\omega}^{\mathrm{FMS}}\right)^{3} \cdot\left|\bar{B}_{\omega}^{\mathrm{FMS}}\right|^{2} \cdot \Delta \omega^{\mathrm{FMS}} \cdot\left(\cos ^{2} \theta_{1}^{\mathrm{FMS}}-\cos ^{2} \theta_{2}^{\mathrm{FMS}}\right)^{-1} \cdot v_{\mathrm{A}}^{4}}{2 \cdot \omega_{b i}^{2} \cdot B^{2} \cdot\left|v_{1}+V_{\|}\right|^{5} \cdot\left(Q^{2}+\eta\right)} \times\left[\frac{\left(v_{\|}+V_{\|}\right)^{2}}{V_{\mathrm{A}}^{2}}-1\right], \\
Q=\frac{\left(v_{\|}+V_{\|}\right)^{2}}{v_{\mathrm{A}}^{2}}-1-\sqrt{\left[\frac{\left(v_{\|}+V_{\|}\right)^{2}}{v_{\mathrm{A}}^{2}}-1\right]^{2}+\eta \cdot \frac{\left(v_{\|}+V_{\sharp}\right)^{2}}{v_{\mathrm{A}}^{2}}} .
\end{array}
$$

\section{A.3. Interaction "proton-beating of A or FMS waves"}

$$
\begin{gathered}
J^{(0)}=0 \quad \alpha=1,2,3 . \\
J_{\alpha}^{(1)}=0 \quad \alpha \\
J_{\alpha \beta}^{(2)}=\delta_{\alpha 3} \delta_{\beta 3} \cdot \frac{T_{i}^{2}}{\left(T_{i}+T_{\mathrm{e}}\right)^{2}} K^{\sigma, \sigma_{1}} . \\
J_{\alpha \beta y}^{(3)}=0 \quad \alpha, \beta, \gamma=0 . \\
K^{\mathrm{A} . \mathrm{A}}=\frac{\chi^{\mathrm{A}, \mathrm{A}}}{56} \cdot\left(W^{\mathrm{A}} / B^{2}\right)^{2} \cdot B^{2} \cdot\left(\bar{\omega}^{\mathrm{A}}\right)^{2} / \Delta \omega^{\mathrm{A}},
\end{gathered}
$$

$K^{\mathrm{FMS} . \mathrm{FMS}}=\frac{\chi^{\mathrm{FMS} . \mathrm{FMS}}}{336} \cdot\left(W^{\mathrm{FMS}} / B^{2}\right)^{2} \cdot B^{2} \cdot\left(\bar{\omega}^{\mathrm{FMS}}\right)^{2} / \Delta \omega^{\mathrm{FMS}}$

$$
\times\left(\cos ^{2} \theta_{\mathrm{I}}^{\mathrm{FMS}}+\cos \theta_{2}^{\mathrm{FMS}} \cos \theta_{1}^{\mathrm{FMS}}+\cos ^{2} \theta_{2}^{\mathrm{FMS}}\right),
$$

$K^{\mathrm{FMS}, \mathrm{A}}=\frac{\chi^{\mathrm{FMS.A}}}{336} \cdot\left(W^{\mathrm{FMS}} \cdot W^{\mathrm{A}} / B^{4}\right) \cdot B^{2} \cdot \bar{\omega}^{2} \cdot \Delta \omega / \Delta \omega^{\mathrm{FMS}} / \Delta \omega^{\mathrm{A}}$

$$
\begin{gathered}
\times\left(3+\cos ^{2} \theta_{1}^{\mathrm{FMS}}+\cos \theta_{2}^{\mathrm{FMS}} \cos \theta_{1}^{\mathrm{FMS}}+\cos ^{2} \theta_{2}^{\mathrm{FMS}}\right), \\
W^{\mathrm{FMS}}=\left|\bar{B}_{\omega}^{\mathrm{FMS}}\right|^{2} \cdot \Delta \omega^{\mathrm{FMS}}, \quad W^{\mathrm{A}}=\left|\bar{B}_{\omega}^{\mathrm{A}}\right|^{2} \cdot \Delta \omega^{\mathrm{A}} .
\end{gathered}
$$

Here, $J^{(0)}, J^{(1)}, J^{(2)}$ and $J^{(3)}$, are the right-hand sides of equations (12), (13), (14) and (15), respectively. 